\title{
Effect of Surface Treatment of Graphite Particles on the Composite Supercapacitor of Hydrous Ruthenium Oxides Coatings by Cathodic Deposition Method
}

Yuli Lin ${ }^{1}$, H.-S Hwang ${ }^{1}$ and Wen-Jin Lee ${ }^{2}$

${ }^{1}$ The Institute of Engineering Science, Chung Hua University, Hsinchu, 300 Taiwan ROC

${ }^{2}$ Material Research Laboratories, Industrial Technology Research Institute

Supercapacitors have many advantages using in electrical devices for their larger capacitance - high power density and long cycle life. In general, supercapacitors can be classified into two categories, namely, pseudo-capacitors and double-layer capacitors. The fomer stores electrical charges in electrode surface region by faradaic reaction. While, in the latter, electrical charges are stored ate the double-layer formed at electrode/electrolyte interface. Various methods have been utilized to manufacture electrode including cyclic voltammetric method 、 sol-gel method 、 anodizing 、 cathodic deposition method and etc.[1].

In this study, hydrous ruthenium oxide with adding graphite particles was deposited on Ti substrate by cathodic deposition method. The purpose of adding graphite particles is to increase the specific surface area of hydrous ruthenium oxide coating. The concentration of graphite particles added in the deposition process varies from $0.1 \mathrm{wt} \%$ to $2 \mathrm{wt} \%$. The effect of surface treatment of graphite particles before deposition was investigated in this study. The graphite particles was treated in 120 ${ }^{\circ} \mathrm{C}-6 \mathrm{M} \mathrm{HNO}_{3}$ solution for one hour to active the graphite surface. The specimen was then immersed into the coating bath for 60 minutes. The electrical capacity characteristics of coating were examined by cyclic voltammetry. The microstructure of hydrous ruthenium oxide coating was also observed by scanning electron microscopy.

Figure 1(a) and (b) show the microstructures of hydrous ruthenium oxide coating after the surface treatment of graphite particles. The electrical capacitance of specimen with $0.3 \mathrm{wt} \%$ of graphite particles after surface treatment was measured about 408F/g. Figure 2 reveals the difference of electrical capacity characteristics of specimens with different concentration of graphite added in deposition processes. Figure 3 reveals the distribution of the capacitance as increasing the concentration of graphite. It can be observed that the capacitance can be increased very effectively when small amount of graphite added in the deposition bath. The reason could be that the graphite after surface treatment will increase the effective area during deposition which leads to the increasing of the amount of hydrous ruthenium oxide. However, it was also found that the 
measured capacitance decreases when the concentration of graphite particles was added more than $0.5 \mathrm{wt} \%$. This is due to the weight of both the hydrous ruthenium oxide and the graphite particles increase which surpass the effective electrical capacity characteristics contributed by hydrous ruthenium oxide coatings.

[1] Yuli Lin and H.-S Hwang, Microscopy and Microanalysis, Vol. 11(suppl.2), p1756-1757(2005).

[2] This research was supported by NSC94-2216-E-216-009, CHU94-2216-E-216-009 and CHU94-TR-09.

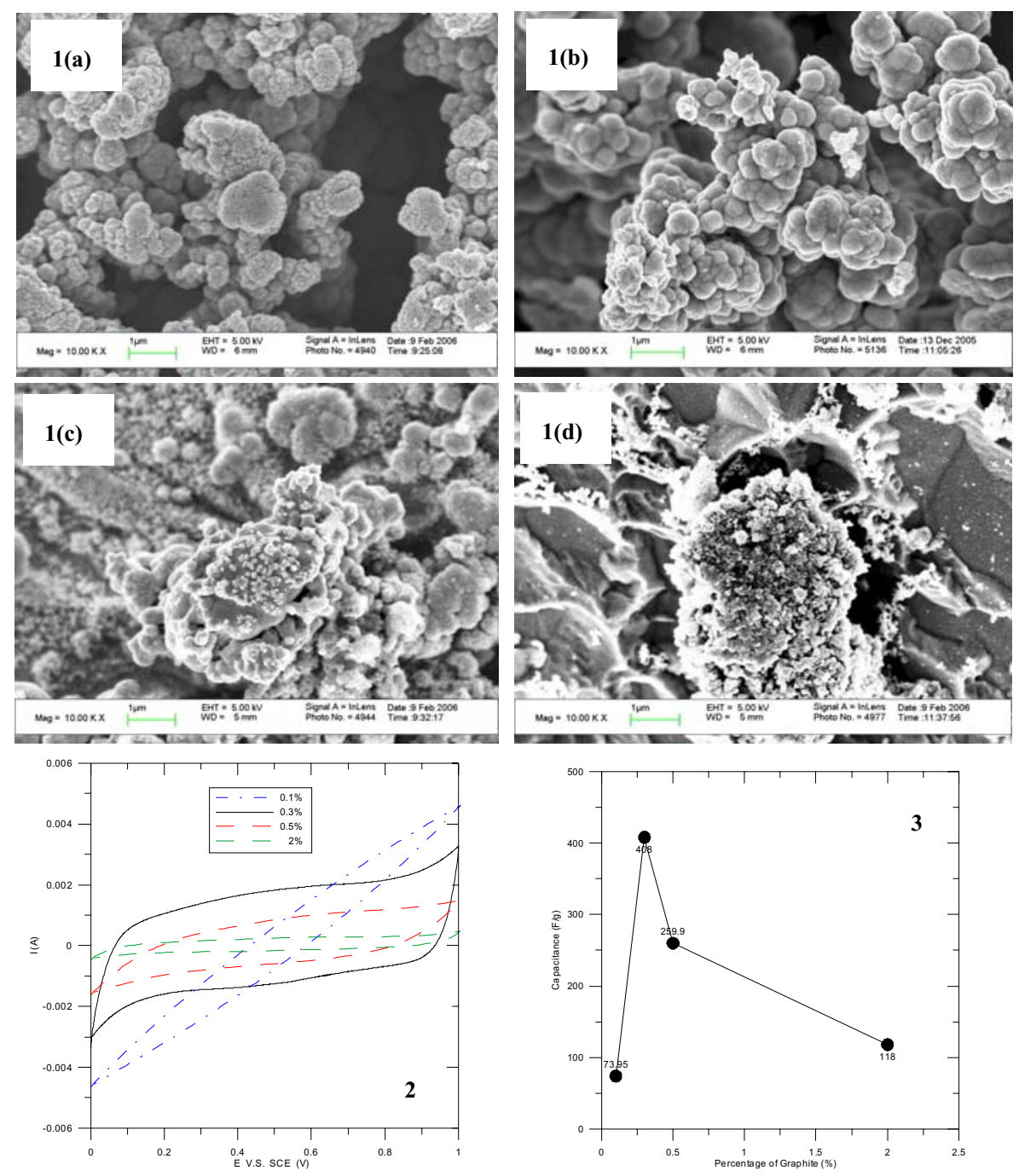

Figure 1. (a)-(d) The microstructure of hydrous ruthenium oxide coating with various graphite particles additives; $0.1 \mathrm{wt} . \%, 0.3 \mathrm{wt} . \%, 0.5 \mathrm{wt} . \%$ and $2 \mathrm{wt} . \%$, respectively.

Figure 2. Eelectrical capacity characteristics of specimens with adding various graphite concentration.

Figure 3. The distribution of capacitance as increasing the concentration of graphite in the deposition processes. 